University of Nebraska - Lincoln

DigitalCommons@University of Nebraska - Lincoln

Combined Kerr microscope and magnetic force microscope for variable temperature ultrahigh vacuum investigations

D. Peterka

Max-Planck-Institute for Solid State Physics

Axel Enders

University of Nebraska-Lincoln, a.enders@me.com

G. Haas

Max-Planck-Institute for Solid State Physics

Klaus Kern

Max-Planck-Institute for Solid State Physics, k.kern@fkf.mpg.de

Follow this and additional works at: https://digitalcommons.unl.edu/physicsenders

Part of the Physics Commons

Peterka, D.; Enders, Axel; Haas, G.; and Kern, Klaus, "Combined Kerr microscope and magnetic force microscope for variable temperature ultrahigh vacuum investigations" (2003). Axel Enders Publications. 11.

https://digitalcommons.unl.edu/physicsenders/11

This Article is brought to you for free and open access by the Research Papers in Physics and Astronomy at DigitalCommons@University of Nebraska - Lincoln. It has been accepted for inclusion in Axel Enders Publications by an authorized administrator of DigitalCommons@University of Nebraska - Lincoln. 


\title{
Combined Kerr microscope and magnetic force microscope for variable temperature ultrahigh vacuum investigations
}

\author{
D. Peterka, A. Enders, ${ }^{\text {a) }}$ G. Haas, and K. Kern \\ Max-Planck-Institute for Solid State Physics, Heisenbergstrasse 1, 70569 Stuttgart, Germany
}

(Received 27 August 2002; accepted 21 January 2003)

\begin{abstract}
A new system combining a Kerr microscope and a magnetic force microscope for the study of magnetic domains in ultrathin films under ultrahigh vacuum conditions is presented. Due to the overlapping imaging range of both techniques magnetic domains can be investigated over a lateral range from millimeter down to fractions of a micrometer. Experiments can be done at variable temperatures, from $80 \mathrm{~K}$ to $600 \mathrm{~K}$. First results are presented showing the same magnetic domain in a three monolayer $\mathrm{Fe}$ film on $\mathrm{Cu}$ (100) imaged with both techniques. (C) 2003 American Institute of Physics. [DOI: 10.1063/1.1568556]
\end{abstract}

\section{INTRODUCTION}

The unique properties of ultrathin magnetic layers and multilayers have attracted wide interest over the past decade. The understanding of the correlation between magnetism and the film structure is the key for tailoring the magnetism in thin films or nanostructures. In particular, in situ domain imaging is of basic interest for the study of the unique magnetic properties of ultrathin films. ${ }^{1}$

Among several imaging techniques for thin film magnetism, scanning electron microscopy with polarization analysis (SEMPA), ${ }^{2,3}$ Kerr microscopy, ${ }^{4}$ and magnetic force microscopy $(\mathrm{MFM})^{5,6}$ are of particular interest due to their high performance. SEMPA offers the advantage of the wide imaging range of a scanning electron microscope for magnetic domain imaging, from millimeters down to several nanometers. This advantage is opposed by the enormous technical effort required for SEMPA experiments. In contrast, Kerr microscopy and MFM can routinely and cheaply be used for magnetic studies. Though, the scan range of these techniques is limited. Furthermore, the quantitative interpretation of the MFM signal is a nontrivial matter and also the influence of the tip stray field may lead to a perturbation of the sample or to different contrast images. ${ }^{7}$ In order to expand the imaging range for magnetic investigations and to obtain complementary information about the magnetic structure, the parallel application of magneto-optical Kerr microscopy and MFM in one single UHV chamber is desired.

Therefore, a new UHV system combining Kerr microscopy, MFM, and scanning tunneling microscopy (STM) for in situ analysis of epitaxial films at variable temperatures was set up and is described in this article. A Besocke-type microscope head ${ }^{8}$ was equipped with a novel in situ tip exchange mechanism which allows one to attach STM or atomic force microscopy (AFM) tips as well as MFM tips to the scan piezo, for structural or magnetic analysis of the films, respectively. This unique combination allows structural and magnetic imaging on the same film in situ. More-

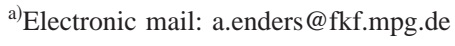

over, investigating the same area on the sample surface by both magnetic imaging techniques is possible. Thus, further investigation of some features in the magnetic structure seen by the Kerr microscope can be done with the enhanced resolution of the MFM. The scan range of the MFM of several micrometer overlaps with the imaging range of the Kerr microscope, thus a direct comparison of both magnetic imaging methods is possible to gain from the complementary information they provide. Also, an extended imaging range from millimeter down to $100 \mathrm{~nm}$ was achieved.

\section{EXPERIMENTAL SETUP}

The magnetic force microscope and the Kerr microscope, together with standard techniques for in situ sample preparation and characterization, are combined in an UHV system shown schematically in Fig. 1. The system consists of a main preparation and analysis chamber (1) and a magnetism chamber (2). A linear manipulator with a travel range of $600 \mathrm{~mm}$ (3) moves the sample between the STM/MFM position in the main chamber and the Kerr position for magnetic investigation. Besides the standard tools for sample preparation, deposition rate calibration, and residual gas analysis, the main chamber is equipped with a Besocke-type STM (4), ${ }^{8}$ for structural characterization of the in situ grown films. The novel tip exchange mechanism allows replacing the STM tip by cantilever tips for AFM of MFM operation. For STM/MFM investigations, the sample holder (5) rests on a viton-damped stack (6) and is mechanically decoupled from the manipulator. After placing the scanning microscope head on the approach ramp, it is also decoupled from its suspension. The microscope, tip-, and sample holder are entirely made of nonmagnetic materials to avoid the presence of any stray field in the vicinity of the sample. The temperature of the sample can be varied either by cooling with liquid nitrogen or by heating with electron bombardment, allowing magnetic and structural investigations in a broad temperature range between $80 \mathrm{~K}$ and $600 \mathrm{~K}$. The temperature is measured by two $\mathrm{Cu}$-constantan thermocouples, which are attached to the bottom of the substrate and the sample holder. The base pressure of the system is $7 \times 10^{-11}$ mbar. 


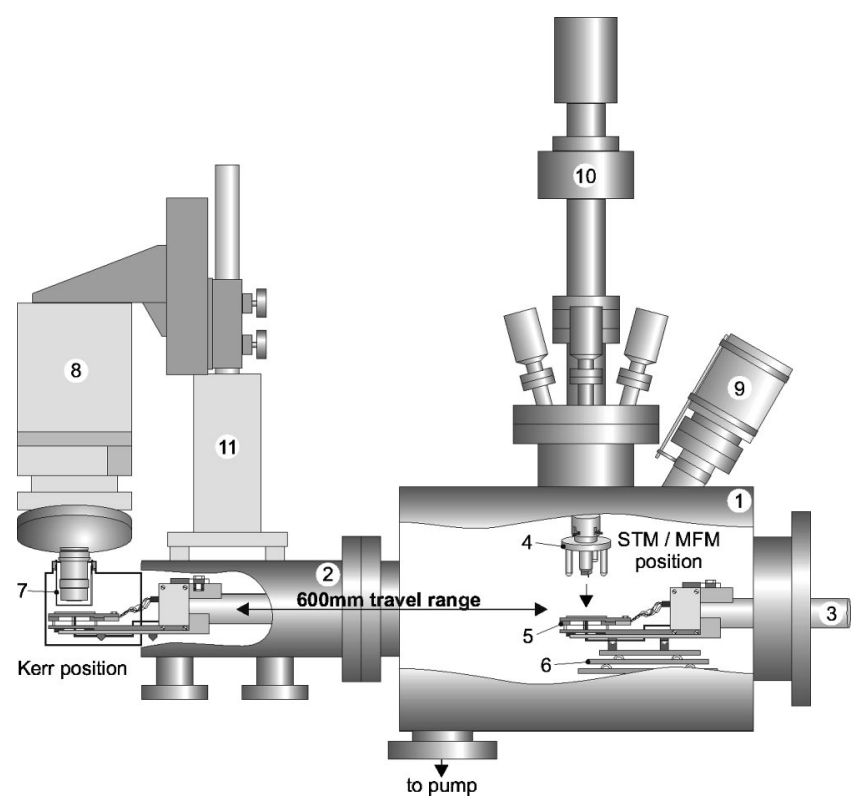

FIG. 1. The UHV system. (1) main chamber, (2) magnetism chamber, (3) long travel range manipulator, (4) STM/AFM/MFM microscope head, (5) sample holder, (6) viton stack, (7) MOKE window, (8) Kerr microscope, (9) optical microscope, (10) manipulator for the scanning microscope, and (11) microscope mount.

The end of the magnetism chamber is only slightly bigger than the sample holder and fits in the gap between the pole pieces of an external electromagnet. Magnetic fields of up to $0.2 \mathrm{~T}$ in polar and in-plane directions of the sample can be applied. The magnetism chamber is equipped with a window (7) designed to accommodate the objective of the Kerr microscope (8), thus allowing a working distance of $<2 \mathrm{~mm}$.

Combined Kerr microscopy and MFM is made possible by a second optical microscope (9) at the MFM position. Specific features seen on the sample surface by the Kerr microscope, e.g., scratches, can be recognized by this second optical microscope. Using such features as markers, the MFM tip can be guided to the desired position on the surface with a precision of $<100 \mu \mathrm{m}$. This allows one to zoom in on sample areas previously imaged with the Kerr microscope.

Next, the design of the beetle-type STM/MFM with its novel in situ tip exchange mechanism and the setup for in situ Kerr microscopy are described in detail.

\section{A. In situ magnetic force microscope with exchangeable tip}

A schematic of the MFM microscope head and the exchangeable tip holder is shown in Figs. 2(a) and 2(b), respectively. The microscope head is based on a beetlelike STM. ${ }^{8}$ In this design the microscope is approached to the sample by three outer piezos moving on a helical ramp by a slip-stick motion. ${ }^{9}$ The instrument is picked up by a suspension tube (I) which is attached to a $x-y-z-\varphi$ translator [(10) in Fig. 1]. In situ MFM requires cantilever deflection measurement compatible with UHV and bakeable up to $150{ }^{\circ} \mathrm{C}$. The MFM/AFM used in this work operates with $\mathrm{Si}_{3} \mathrm{~N}_{4}$ piezoresistive cantilevers with a typical force constant of $20 \mathrm{~N} / \mathrm{m} .{ }^{10}$ Cantilever deflections due to tip-sample interaction result in the change of the electrical resistance of the cantilever and


FIG. 2. (a) Beetle-type microscope head with socket for the tip, (b) exchangeable tip holder; the attachment of the AFM tip to the tip holder is shown enlarged, and (c) the tip holder is attached to the scan piezo by plugging in the chinch connector and rotating the microscope head to tighten the thread. (1) Electrical contact, (2) Ti body, (3) spring contact, (4) Ti nut, $(5,8,10)$ Macor spacer, (6) female contact with inner thread, (7) Ti pin, (9) Ti contact with thread, (11) Ti body, and (12) piezoresistive cantilever with contact pads.

can easily be measured with a Wheatstone bridge. Thus, no optical or other alignment, which can conveniently be done in ambient systems but is difficult to realize under UHV, ${ }^{11-13}$ is necessary. Therefore, tip replacement is also relatively easy.

Here, we describe the new design of the in situ tip exchange mechanism. The mechanical and electrical connections between the scan piezo of the microscope head in Fig. 2(a) and the tip holder in Fig. 2(b) were inspired by a common audio stereo jack (cinch connector). The tip holder is equipped with a $\mathrm{Ti}$ pin (7) and an outer $\mathrm{Ti}$ contact with thread (9). Both contacts are electrically insulated from each other by macor spacers $(8,10)$. The female counterpart on the bottom end inside the scan piezo of the microscope head consists of a Ti contact with inner thread (6) and a spring contact (3). Both parts are wired to a socket to allow output of the electrical signals. In order to attach the tip holder to the microscope head, the tip holder is simply plugged into the opening (6), followed by a half turn of the microscope head to tighten the screw mechanism. Thus, mechanical stability is enhanced substantially as is necessary for the excitation of self-oscillation of the cantilever in noncontact mode AFM and MFM. Pin (7) and contact spring (3), as well as contacts (9) and (6), establish the electrical connection.

The cartridge containing the piezoresistive cantilever and contact pads for electrical connection is clamped on the bottom of the tip holder (12), as shown more detailed at the bottom of Fig. 2(b). The piezoresistive cantilever is mounted at the front end of a thin ceramics chip (white). The cantilever is electrically connected to the contact pads on both sides of the ceramic chip. Clamping the cartridge establishes the electrical connection of the contact pads to the Ti body and the spring clamp, and thus to the cinch connector. The cartridge can be replaced by a tunneling tip, which is then mounted to the Ti body (11) directly. The scan piezo itself has to be large enough to accommodate the connector for the tip holder. With the scan piezo used a scan range of $10 \mu \mathrm{m} \times 10 \mu \mathrm{m}$ can be achieved.

The tip exchange is schematically shown in Fig. 2(c). Spare tips are stored in situ in a probe stock (III), which can 
be moved by a rack and pinion linear manipulator. After positioning the tip below the microscope head (II), the microscope is lowered to pick up the tip holder and to establish the mechanical and electrical connections. With a half turn of the microscope head by the microscope suspension (I), the screw joint is tightened to improve the stiffness of the mechanical connection.

For magnetic imaging, the piezolever tips were coated with $30 \mathrm{~nm}$ Co and passivated with $5 \mathrm{~nm}$ Au in a different chamber. Prior to loading into the UHV system the tips are magnetized along the tip normal and outgassed in the load lock. For noncontact AFM/MFM operation the frequency modulation technique introduced by Albrechts et al. ${ }^{14}$ is used. The cantilever is driven at its eigenfrequency at constant amplitude. Changes in its vibration frequency due to changes in the force gradient acting on the tip are detected. These force gradients are mainly due to van der Waals interactions for tip-sample distances in the $\mathrm{nm}$ range or magnetic forces (or electrostatic forces) for distances in the order of $100 \mathrm{~nm}$.

Magnetic images can be recorded in two different ways: (i) The slope between sample and imaging plane is determined first by taking a line scan in $x$ and $y$ directions outside the area of interest with the tip approached to the surface. Then, during imaging, the feedback loop is switched off and the tip is somewhat retracted. Scanning in constant height mode is now realized by following this estimated slope. The measured shift in the resonance frequency is translated by a band-pass filter and a phase comparator into a phase shift signal and represents the force gradient of the long ranging magnetic force. (ii) The oscillating tip scans across the sample surface with the frequency shift kept constant by the feedback loop. In this mode, the obtained height $(z)$ signal represents the surface of constant force gradient. An additional voltage $(0-10 \mathrm{~V})$ has to be applied to the electrically insulated sample to stabilize the servoloop and to avoid snapping in of the tip.

\section{B. In situ Kerr microscopy}

Only a few approaches have been done in the past to adapt Kerr microscopy to UHV systems. For instance, an in situ Kerr microscope with a high-pressure mercury lamp as an illumination source and a long distance microscope for sample imaging has been built by Kirschner et al. ${ }^{15} \mathrm{~A}$ somewhat different approach was used by Vaterlaus et al. ${ }^{16}$ where a scanning Kerr Microscope was realized using a highly focused laser diode.

The Kerr Microscope developed in this work is based on a commercially available Zeiss polarization microscope, ${ }^{17}$ which is known to give excellent magnetic contrast but was applied so far only to ambient systems. The microscope is equipped with a Nikon microscope mount ${ }^{18}$ and attached to a solid tripod directly on the chamber to improve the image stability [(11) in Fig. 1]. This flexible joint allows easy optical alignment, removal of the microscope for chamber bake outs, and replacement by an optical bench for conventional magneto-optical Kerr effect (MOKE) measurements. To image domains, the microscope is lowered by its mount until

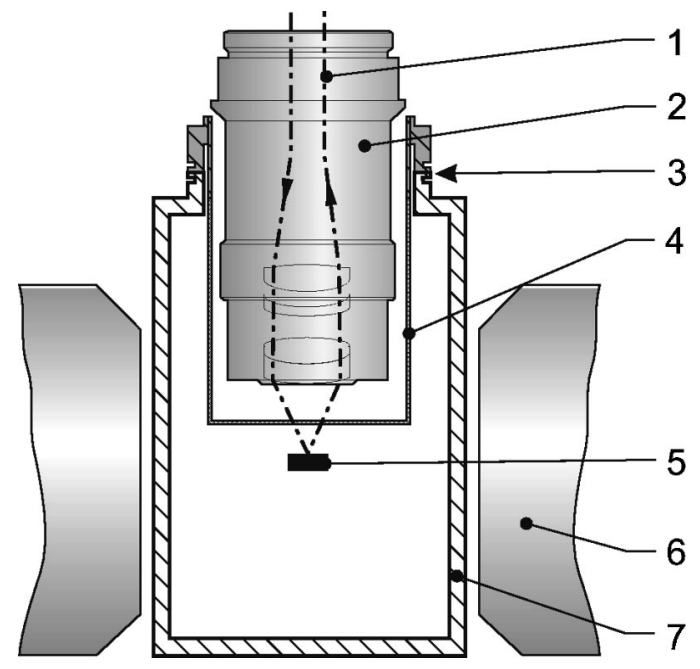

FIG. 3. The design of the UHV window for Kerr microscopy allows for objective-sample distances of $2 \mathrm{~mm}$. (1) Laser beam, (2) objective of the microscope, (3) window-chamber-joint, (4) strain-free quartz glass window, (5) sample, (6) magnet yoke, and (7) UHV chamber.

the objective lens is just above the flat bottom of the window of the magnetism chamber, as shown in Fig. 3. The particular design of the window allows one to operate at a minimum sample-lens distance and to use microscope objectives with a working distance as short as $2 \mathrm{~mm}$ !

For magnetic imaging with the Kerr microscope, the sample has to be transferred in situ into the magnetism chamber - a nonmagnetic stainless-steel cuboid welded to a DN100CF flange and mounted at the end of the chamber. This chamber is only $50 \mathrm{~mm}$ wide and fits between the iron yoke of an external electromagnet. Magneto optics is done on the sample through a specially designed viewport ${ }^{19}$ allowing a short working distance between the objective of the microscope and the sample surface (Fig. 3). A distortion-free and strain-free quartz glass tube with a molybdenum quartz seal is welded to a stainless-steel adapter (3) on the magnetism chamber. On the flat bottom of the window, an antireflective coating ${ }^{20}$ was deposited to avoid image quality loss due to backscattered light from the window itself.

To reach high optical resolution, a setup suggested by Hubert was adopted. ${ }^{4}$ The illumination source, the polarizer, and analyzer mounts as well as the support was adapted to fit the needs of Kerr microscopy and the UHV apparatus. The beam splitter has been replaced by a Berek prism. GlanThompson polarizers were used to achieve an extinction ratio of less than $2 \times 10^{-5}$. A $1 \mathrm{~W}$ Ar-ion laser is used as a light source to supply monochromatic, stabilized, high intensity illumination. By using a laser instead of commonly used high-pressure mercury lamps ${ }^{15}$ problems with limited stability and lifetime were eliminated. The laser light is coupled into a $200 \mu \mathrm{m}$ quartz-quartz multimode optical fiber (MMF) with a numerical aperture of NA=0.20. Because of the small diameter of the light beam, no aperture stop is needed. Speckle patterns in the image due to interference effects of the coherent light are removed by mechanically agitating several meters of the MMF at its eigenfrequency of the order of $\sim 100 \mathrm{~Hz}$. 
To attain uniformly illuminated high-quality Kerr images of the specimen with homogeneous extinction across the entire field of view, well-adjusted reflection Köhler illumination is necessary. This is easily achieved by optimizing the MMF tip position along the optical axis of the microscope. On the other hand, the position of the image of the light guide within the objective back focal plane, and thus the angle and direction of incidence of the light onto the surface, is adjusted by moving the MMF tip perpendicular to the optical axis. All lateral and angular alignments of the fibre exit with respect to the microscope are carried out by micrometer screws. Adjustment of internal lenses, as needed with Kerr microscopes using a slit aperture diaphragm ${ }^{4}$ is not needed.

The angle of incidence can be varied between $0^{\circ}$ and $15^{\circ}$, making possible longitudinal, polar, and transversal Kerr geometries. A long-distance objective lens with a magnification of mag $=16 \times$ and NA $=0.2$ is mostly used. With this setup an optical resolution of approximately $1 \mu \mathrm{m}$ is reached. Images of $400 \times 300 \mu \mathrm{m}^{2}$ were captured with a charge coupled device (CCD) camera. ${ }^{21}$ Data acquisition with a maximum repetition rate of 5 s/image was done by a real-time digital image processor. ${ }^{22}$

\section{RESULTS}

To demonstrate the capability of the combined Kerr microscope/Magnetic force microscope, as-grown magnetic domains of $3 \mathrm{ML} \mathrm{Fe}$ on $\mathrm{Cu}(001)$ were investigated. The film was grown at $130 \mathrm{~K}$ by molecular beam epitaxy and imaged at the same temperature. MFM images were obtained in the constant height mode following the slope of the sample surface determined prior to the image acquisition. The MFM images in Figs. 4(b)-4(e) correspond to the shift of the cantilever oscillation frequency during scanning.

Before film preparation the $\mathrm{Cu}(001)$ single crystal substrate was cleaned by different cycles of $500 \mathrm{eV} \mathrm{Ar}^{+}$-ion sputtering followed by annealing up to $830 \mathrm{~K}$, as described in Ref. 23. The final annealing was done at $650 \mathrm{~K}$, followed by controlled cooling-down to RT with a rate of $2 \mathrm{~K} / \mathrm{s}$. This procedure was found to maximize the terrace width. ${ }^{24}$ The surface quality was checked afterward by STM. Fe was evaporated from a high-purity Fe rod by electron beam heating. The deposition rate of $0.4 \mathrm{ML} / \mathrm{min}$ was calibrated by STM and kept constant during evaporation by manually adjusting the acceleration voltage.

Three monolayers (MLs) of $\mathrm{Fe}$ on $\mathrm{Cu}(001)$ show an easy axis of magnetization perpendicular to the sample surface. ${ }^{25}$ At this low growth temperature of $130 \mathrm{~K}$, a multidomain pattern, as shown in the Kerr image in Fig. 4(a), is developed in the film. ${ }^{26}$ The dark/bright contrast is due to magnetic domains of opposite magnetization perpendicular to the plane. The Kerr images in Figs. 4(a) and 4(f) are taken without background subtraction. Thus, defects on the sample surface are visible and marked by white arrows in the images. These defects are used to guide the MFM tip to the desired region on the sample surface under the control of the second optical microscope. The same magnetic domain, as highlighted in the Kerr image in Fig. 4(a), can be found by MFM



FIG. 4. The same magnetic domain imaged under UHV by [(a) and (f)] Kerr microscopy and [(b)-(e)] MFM. Changes in the domain configuration caused by the dipolar field of the MFM tip can be traced by the Kerr microscope afterward (f). From (b) to (e), the MFM tip was approached in $20 \mathrm{~nm}$ steps.

and is further investigated with the enhanced resolution of the MFM [Fig. 4(b)]. The domain wall separating two regions of perpendicular magnetization is clearly visible in the center of the image in Fig. 4(b).

After acquisition of the MFM image in Fig. 4(b) the distance between the sample and MFM tip was gradually reduced by $20 \mathrm{~nm}$ steps [Figs. 4(b)-4(e)]. With decreasing distance, the dipolar field of the magnetic tip became strong enough to drag the domain wall during scanning. The MFM image in Fig. 4(c) already shows the domain wall slightly displaced toward the left-hand side border of the image. Finally, in Fig. 4(e) the domain is driven out of the imaged region. The Kerr image in Fig. 4(f) subsequently taken after the MFM studies on the same spot, clearly shows these alterations. Again, this area was found by characteristic features on the sample surface. Also visible in Fig. 4(f) are other changes in the domain pattern due to previous MFM scans on different positions.

The magnetic signal in Figs. 4(b)-4(e) corresponds to a cantilever frequency shift of $50 \mathrm{mHz}$ due to the magnetic interaction with the $3 \mathrm{MLs}$ thin Fe film. The signal is mainly limited by the spring constant of the cantilever. Improvements will be achieved by using tips of smaller spring constant. The lateral resolution of the MFM is determined on the $180^{\circ}$ domain wall of a iron whisker and found to be better than $150 \mathrm{~nm}$.

In conclusion, a combined Kerr microscope and MFM for in situ domain imaging was described. The shown ex- 
ample proves that combined Kerr/MFM studies on the same magnetic feature are possible. The combination of both techniques covers a lateral range extending from $\geqslant$ hundred nanometer up to millimeter. It delivers complementary information and allows one to image local modifications of the domain structure. Furthermore, a novel tip exchange mechanism was presented which enables the microscope to be operated as an STM, AFM, and MFM. To achieve this, the tips can conveniently be replaced in situ with no need for breaking the vacuum.

\section{ACKNOWLEDGMENT}

The authors thank H. U. Habermeier for the expert magnetic coating of the piezoresistive cantilevers.

${ }^{1}$ M. R. Freeman and B. C. Choi, Science 294, 1484 (2001).

${ }^{2}$ H. P. Oepen and J. Kirschner, Scanning Microsc. 5, 1 (1991).

${ }^{3}$ M. R. Scheinfein, J. Unguris, M. H. Kelley, D. T. Pierce, and R. J. Celotta, Rev. Sci. Instrum. 61, 2501 (1990).

${ }^{4}$ A. Hubert and R. Schäfer, Magnetic Domains (Springer, Heidelberg, 2000).

${ }^{5}$ Y. Martin and H. K. Wickramasinghe, Appl. Phys. Lett. 50, 1455 (1987).

${ }^{6}$ J. Sáenz, N. Garcia, P. Grütter, and E. Meyer, J. Appl. Phys. 62, 4293 (1987).

${ }^{7}$ M. Schneider, S. Müller-Pfeiffer, and W. Zinn, J. Appl. Phys. 79, 8578 (1996).
${ }^{8}$ K. Besocke, Surf. Sci. 181, 145 (1987).

${ }^{9}$ J. Frohn, J. F. Wolf, K. Besocke, and M. Teske, Rev. Sci. Instrum. 60, 1200 (1989).

${ }^{10}$ Autoprobe VP piezolevers, Park Scientific Instruments, Sunnyvale, CA.

${ }^{11}$ A. Wadas, M. Dreyer, M. Kleiber, and R. Wiesendanger, Appl. Phys. A: Mater. Sci. Process. 66, 465 (1998).

${ }^{12}$ Omicron Vacuumphysik GmbH, Limburger Str. 75, 65232 Taunusstein, Germany.

${ }^{13}$ U. Memmert, P. Leinenbach, J. Lösch, and U. Hartmann, J. Magn. Magn. Mater. 190, 124 (1998).

${ }^{14}$ T. Albrecht, P. Grütter, D. Horne, and D. Rugar, J. Appl. Phys. 69, 668 (1991).

${ }^{15}$ J. Giergiel and J. Kirschner, Rev. Sci. Instrum. 67, 2937 (1996).

${ }^{16}$ A. Vaterlaus, U. Maier, U. Ramsperger, A. Hensch, and D. Pescia, Rev. Sci. Instrum. 68, 2800 (1997).

${ }^{17}$ Jenapol polarizing microscopes, Zeiss Jena GmbH, Jena, Germany.

${ }^{18}$ Nikon GMBH, Düsseldorf, Germany, Modular Focusing Unit MBD64000.

${ }^{19}$ Bomco Inc. 125 Gloucester Avenue, Gloucester, MA 01930-2254.

${ }^{20}$ Leica Microsystems AG, Glattbrugg, $\mathrm{CH}$, antireflective coating ARB2.

${ }^{21}$ CF 8/1 CCD video camera, Kappa Messtechnik GmbH, Gleichen, Germany.

${ }^{22}$ HAMAMATSU, real time digital image Processor ARGUS20.

${ }^{23}$ J. Thomassen, B. Feldmann, and M. Wuttig, Surf. Sci. 264, 406 (1992).

${ }^{24}$ J. Giergiel, J. Kirschner, J. Landgraf, J. Shen, and J. Woltersdorf, Surf. Sci. 310, 1 (1994).

${ }^{25}$ R. Allenspach and A. Bishof, Phys. Rev. Lett. 69, 3385 (1992).

${ }^{26}$ D. Peterka, A. Enders, G. Haas, and K. Kern, Phys. Rev. B 66, 104411 (2002). 